\title{
On optimal designing of low frequency polychromatic fields for facile photo-dissociation of model diatomic molecules ${ }^{\dagger}$
}

\author{
S GHOSH, K MAJI, R SHARMA and S P BHATTACHARYYA* \\ Department of Physical Chemistry, Indian Association for the Cultivation of Science, Jadavpur, \\ Kolkata 700032 \\ e-mail: pcspb@mahendra.iacs.res.in
}

\begin{abstract}
The dissociation of a diatomic molecule in low frequency polychromatic fields of moderate intensities is studied. Genetic Algorithm is invoked to search out a set of four optimal non-resonant frequencies $\left(\omega_{1}-\omega_{4}\right)$, intensities $\left(\varepsilon_{1}-\varepsilon_{4}\right)$ the and phase angles $\left(\delta_{1}-\delta_{4}\right)$, for achieving a facile photo dissociation. Time-dependent Hellmann-Feynman theorem is used to gain insight into the frequency resolved energy absorption pattern. The 'quantum phase space' structures indicate occurrence of bond breaking dynamics akin to the classical one.
\end{abstract}

Keywords. Photodissociation; TDFGH; genetic algorithm; time dependent Hellmann-Feynmann theorem; quantum phase space.

\section{Introduction}

The response of free atoms and molecules in strong radiation field has attracted a lot of theoretical and experimental attention in the recent years. The theoretical interest primarily stems from the fact that the interaction between matter and radiation in strong field regimes poses questions that are yet to be answered fully. On the experimental front the problem of realizing appropriate laser intensity, frequency, pulse shape and duration required for the emergence of observable strong field effects, tend to raise fundamental questions concerning absorbability of such effects. Nevertheless, efforts are on for understanding and solving the problems both at the theoretical and experimental levels. Thus photo electron spectroscopy of neutral atoms in strong fields has detected above threshold ionization peaks, ${ }^{1,2}$ while emission spectroscopy of neutral atoms in intensity regimes where nonlinear atomic response becomes appreciable, has revealed the appearance of new frequencies at higher multiples of the driving frequencies. ${ }^{3}$ It is conceivable that similar phenomena can be observed in molecular multi-photon excitations. ${ }^{4}$ There have been studies on the photo fragmentation of small molecular systems in intense laser fields where the dynamics occurs on rather short time

\footnotetext{
${ }^{\dagger}$ Dedicated to the memory of the late Professor S K Rangarajan
} *For correspondence scales $\leq 10 \mathrm{fs}$ raising questions pertaining to the expected behaviour of molecular vibrations under intense laser irradiations. ${ }^{5,6}$ The dynamics of a periodically driven Morse oscillator has been a good model for understanding the behaviour of molecular vibrations under the influence of continuous irradiation. ${ }^{7-10}$ It has been demonstrated through numerical experiments that at intensities $<10^{14} \mathrm{~W} / \mathrm{cm}^{2}$ the dissociation probability $\left(P_{d}\right)$ is less than $10^{-5}$ when monochromatic continuous sub-picosecond pulsed IR laser is used. ${ }^{6}$ The anharmonicity of the vibrational potential has been known to be responsible for the low dissociation probability at such intensities.

Photo-dissociation takes place when the energy eigen-states reached by the photo absorption are in the continuum. From an experimental point of view, the control of a photo-dissociation event requires light sources that generate $\left(\omega_{1}, \omega_{2}, \omega_{3}, \omega_{4}\right.$, etc. $)$ frequencies. The sources may be pulsed or continuous wave lasers. Nonlinear theory of classical Hamiltonian systems has been known to predict phase dependence of photo dissociation probability when two laser intensity sources are used. ${ }^{11,12}$ If the first laser is kept constant just above the single laser dissociation threshold, the addition of a second laser suppresses the dissociation indicating, as if, bond hardening has taken place. The bond hardening diminishes as the phase difference between the two lasers increases. It has been argued from the classical nonlinear dynamical point of view that variations 
in phase and intensity may cause appearance or disappearance of stability islands corresponding to the common resonance with the lowest energy deformation, and movement of the region of KolmogorovArnold-Moser Tori that survive from the undriven system. The second is supposed to be the main origin of the phase space stabilization and phase dependence. $^{13,14}$ The question of correspondence between the classical results and the quantum mechanical analogue largely remains to be probed.

If the single laser has a frequency much below the dissociation threshold, a bottle-necking to dissociation is usually observed unless the intensity is very high. The origin of the bottle-necking here lies in vibrational anharmonicity. The anharmonicity bottleneck to dissociation can be overcome with appropriately chirped pulses in which the frequency is so modulated that the anharmonicity in the spacings of the particular vibration is matched. ${ }^{5,6}$ At higher intensities the coupling between the field and the oscillator may be strong enough to create dressed states of the oscillator completely obliterating the anharmonicity bottle neck. The dissociation rate, in fact has strong non-linear dependence on the intensity of the radiation. Another way of bypassing the bottle-necking problem is multi-photon dissociation of a diatomic species using a bi-chromatic field with controlled phase difference. ${ }^{15,16}$ The use of polychromatic fields with carefully chosen frequencies, intensities and phases, may modify the underlying structures in the phase space and thereby influence dissociation probabilities and rates favourably.

With this background in view, we propose to investigate the photo-dissociation dynamics of a hetero diatomic molecule in continuous IR laser fields with four different frequencies $\left(\omega_{1}, \omega_{2}, \omega_{3}, \omega_{4}\right)$ none of which are 'on-resonance' or have 'above threshold' intensity. The focus of the investigation has been: (i) to explore the dependence of photodissociation rate and probability on frequency ratio; (ii) to find out the best set of four non-resonant frequencies and the corresponding below threshold intensities that lead to faster and more complete dissociation; (iii) to find out the frequency resolved energy gain pattern; (iv) to understand the nature of the bond stretching dynamics and the motion of the system in the so called 'quantum phase space'with a view to asserting is there any signature of bond hardening?

We carry out the proposed calculations in the framework of time-dependent Fourier Grid Hamil- tonian method and use the time-dependent Hellmann-Feynman theorem to partition the total energy input over component frequencies. Floating point Genetic Algorithm ${ }^{17-20}$ has been used to search out the optimal frequency ratio, intensities and phase angles for different components of the polychromatic field.

\section{Methodology}

\subsection{The TDFGH formulation}

The TDFGH method ${ }^{21-23}$ follows quite easily and naturally when the time-dependent variational recipe is invoked within the framework of the $\mathrm{FGH}^{24,25}$ method. We can describe of a diatomic moleculepoly-chromatic field system, by a one-dimensional time-dependent Hamiltonian $H(x, t)$ where,

$$
H(x, t)=T(x)+V^{\ominus}(x)+V(x, t),
$$

for linear coupling (no electrical anharmonicity)

$$
V(x, t)=\sum_{i=1}^{n} x|e| \varepsilon_{i} \sin \left(\omega_{i} t+\delta_{i}\right)
$$

and

$$
V_{1}(x, t)=\sum_{i=1}^{n} f(x) x|e| \varepsilon_{i} \sin \left(\omega_{i} t+\delta_{i}\right),
$$

where

$$
f(x)=e^{-\left(x-x_{e}\right)^{2} / b},
$$

for a Gaussian type of coupling (electrical harmonicity is present) $b$ in (4) is so chosen that the dipole moment displays maximum around $v=8-10$ before falling to zero value as $x \rightarrow \infty$. So the timedependent Hamiltonian describing our system can be represented as

$$
\begin{aligned}
H(x, t) & =T(x)+V^{0}(x)+V(x, t) \text { or } V_{1}(x t), \\
& =h^{0}(x)+V(x, t) \text { or } V_{1}(x, t),
\end{aligned}
$$

where $V^{0}(x)$ is the appropriate Morse potential describing the diatomic species. $\varepsilon_{i}$ is the intensity of the electric field of the radiation of frequency $\omega_{i}$ and phase $\delta_{i}$ and $n$ is the number of component frequen- 
cies present. At $t=0$, the initial states are eigenstates $\phi_{i}^{0}(x)$ of the isolated Morse oscillator Hamiltonian $h^{0}(x)$ while for $t>0$ the wave function $\psi(x, t)$ can be described by a time-dependent superposition of the unperturbed eigenstates of the Morse oscillator, i.e.

$$
|\psi(x, t)\rangle=\sum_{i} a_{i}(t)\left|\phi_{1}^{0}(x, t)\right\rangle
$$

where $\left|\phi_{i}^{0}(x)\right\rangle=\sum_{p=1}^{n_{x}}\left|x_{p}\right\rangle \Delta x$ in the FGH representation $^{24,25}$ of the unperturbed function. In TDFGH ${ }^{21-23}$ method we represent $|\psi(x, t)\rangle$ on a uniformly discretized grid (spacing $\Delta x$, length $l$, no. of grid points $n_{x}$ ) with time-dependent grid point amplitudes as follows

$$
|\psi(x, t)\rangle=\sum_{p}^{n_{x}} w_{p}(t)\left|x_{p}\right\rangle \Delta x
$$

with the orthogonality conditions on the grid specified as

$$
\left\langle x_{p} \mid x_{q}\right\rangle \Delta x=\delta_{p q} .
$$

Application of Dirak-Frenkel variational princi$\mathrm{ple}^{26,27}$ then leads to the evolution equations of the grid point amplitudes $w_{p}(t)$ as follows

$$
i \hbar \dot{w}_{p}(t)=\sum_{q=1}^{n_{x}}\left\langle x_{p}|H(x, t)| x_{q}\right\rangle w_{q}(t), p=1,2, \ldots n_{x} .
$$

These equations can be numerically integrated once values of $w_{p}(t=0)$ are provided. For the optimization of field parameters the dissociation probability at a given instant is computed without the imposition of absorbing boundary condition as follows. We compute the generalized overlap amplitudes of $|\psi(x, t)\rangle$ with all the $n_{b}$ numbers of bound states of the unperturbed Morse oscillator. The dissociation probability at time $t$ is given by

$$
P_{d}(t)=1-\sum_{i=0}^{n_{b}} s_{i}^{2}(t)
$$

where $s_{i}=\left\langle\varphi_{i}^{0} \mid \psi(x, t)\right\rangle$. Therefore,

$$
P_{d}(t)=1-\sum_{i=0}^{n_{b}}\left|\left\langle\phi_{i}^{0} \mid \psi(x, t)\right\rangle\right|^{2},
$$

$P_{d}$ grows in time with oscillations and the slope of $\ln P_{d}(t)$ with respect to $t$ gives the instantaneous dissociation rate constant $K_{d}(t)$. Computed this way may still $K_{d}(t)$ have a lot of oscillations. For a more meaningful presentation of dissociation rate constant $\ln P_{d}(t)$ may be averaged over a number of time steps $(t=n \Delta t, n=1000$, typically)

$$
\bar{P}_{d}(t)=\frac{1}{t} \int_{0}^{t} P_{d}\left(t^{\prime}\right) \mathrm{d} t^{\prime}
$$

\subsection{The genetic algorithm used for optimization}

$\bar{P}_{d}(t=T)$ is expected to be a function of the component frequencies $\left(\omega_{1}-\omega_{4}\right)$, the corresponding intensities $\left(\varepsilon_{1}-\varepsilon_{4}\right)$ and the phase angle $\delta_{1}-\delta_{4}$. The parameters are optimized by a floating point genetic algorithm developed by us. ${ }^{28}$ The strings $S_{k} \equiv$ $S\left(\omega_{1}^{k}, \omega_{2}^{k}, \omega_{3}^{k}, \omega_{4}^{k}, \varepsilon_{1}^{k}, \varepsilon_{2}^{k}, \varepsilon_{3}^{k}, \varepsilon_{4}^{k}, \delta_{1}^{k}, \delta_{2}^{k}, \delta_{3}^{k}, \delta_{4}^{k}\right) \quad$ are one-dimensional arrays containing all the information about the four component field. The constraints on the variables are the following:

$$
\begin{aligned}
& 0 \cdot 75 \hbar \omega_{01} \leq \hbar \sum_{i} \omega_{i}^{k} \leq 1 \cdot 25 \hbar \omega_{01}, \\
& \sqrt{\sum_{i} \varepsilon_{i}^{2}}=\varepsilon_{0}, \\
& -2 \pi \leq \delta_{i} \leq 2 \pi, \quad i=1,2,3,4 .
\end{aligned}
$$

The fitness function of the $k$ th string (which is sought to be maximized) is defined as follows:

$$
f_{k}=e^{-\pi(\lambda)^{2}}
$$

where $T$ is the time required to reach the value $P_{d}(T)=0.8$ (see (12) for the definition of $P_{d}(T)$ and $\lambda$ is a scalar chosen by the user. Roulette wheel selection with arithmetic crossover, mutation, diversification $^{28}$ on a population of size $N_{p}=10$ has been used. The crossover probability $p_{c}$, mutation probability $p_{m}$, and diversification probability $p_{d}$ have been kept fixed at $0.80,0.40$ and 0.04 , respectively. The mixing amplitude for crossover has been set at $f_{c}=0.75 \pm 0.25$ depending on whether the random number $r(0<r<1)$ generated at the crossover stage is less than or greater than 0.5 . The mutation intensity $f_{m}$ and the diversification intensity $f_{d}$ have been 
allowed to vary dynamically following the scheme outlined below.

Let $N_{f}$ be the number of offsprings in a generation with the maximum fitness value. If $N_{f} / N_{p}<0 \cdot 1$ we reset either the mutation or diversification intensity $f_{m}=0.9 * f_{m}$ or $f_{d}=1.1 * f_{d}$, randomly while if $N_{f} / N_{p} \geq 0 \cdot 3$, we reset the same parameters with either $f_{m}=1 \cdot 1 * f_{m}$ or $f_{d}=0 \cdot 9 * f_{d}$, randomly. If $f_{m} \geq 10$ or $f_{d} \leq 10^{-10}$, the process is terminated. After selection, crossover, mutation or diversification, 50 percent of the offsprings are chosen on the basis of elitist screening while the remaining 50 percent are chosen randomly.

Once the optimal parameter sets are found, we do a final calculation of $P_{d}(t)$ and the dissociation rate by using the absorbing boundary condition. ${ }^{29}$ The rate of the decrease of the norm $N(t)$ of the wave function directly gives the dissociation rate while $N(t) / N(0)$ gives $P_{d}(t)$, the dissociation probability at time $t$.

The energy $E$ delivered to the oscillator by the radiation of frequency $\omega_{i}$ is computed from timedependent Hellman-Feynman theorem as follows

$$
\begin{aligned}
& \frac{d(E)}{\mathrm{d} t}=\left\langle\psi(x, t)\left|\frac{\mathrm{d} H}{\mathrm{~d} t}\right| \psi(x, t)\right\rangle \\
& =\sum_{i=1}^{n}|e| \varepsilon_{i} \omega_{i} \cos \left(\omega_{i} t+\delta_{i}\right)\langle\psi(x, t)|f(x) x| \psi(x, t)\rangle \\
& =\sum_{i=1}^{n}|e| \varepsilon_{i} \omega_{i} \cos \left(\omega_{i} t+\delta_{i}\right)\langle f(x) x\rangle_{t},
\end{aligned}
$$

where

$$
\langle f(x) x\rangle_{t}=\langle\psi(x, t)|f(x) x| \psi(x, t)\rangle .
$$

Average energy delivered over the time span $t$ at frequency $\omega_{i}$ one optical cycle at frequency $\omega_{i}$ is

$$
\bar{\varepsilon}_{i}(t)=\frac{1}{t}|e| \omega_{i} \varepsilon_{i} \int_{0}^{t} \cos \left(\omega_{i} t^{\prime}+\delta_{i}\right)\langle f(x) x\rangle_{t} \mathrm{~d} t^{\prime} .
$$

The integration is done numerically. The average numbers of photons of frequency $\omega_{i}$ absorbed over a time span of $t$ is given by

$$
\overline{n_{i}(t)}=\frac{\overline{\varepsilon_{i}(t)}}{\hbar \omega_{i}} .
$$

Equation (18) and (19) are used to compute the frequency resolved energy gain pattern of the molecule along with the number of photons absorbed.

\section{Results and discussion}

We have initially experimented (numerically) with different two, three and four component fields. It turns out that optimal designing works best for a four component polychromatic field. No significant effects of designing could be seen with two or three component fields. We therefore propose to present in this communication the results pertaining to four component fields only. The molecule studied is lithium hydride which is modelled by an appropriate Morse oscillator (table 1). The TDFGH calculations have been carried out on a grid of length 30 a.u. with an absorbing potential located at $x_{c} \geq 20$. Sixth order Runge Kutta method has been used to integrate the time-dependent Schrödinger equation with a time step of 0.025 a.u.

The dissociation threshold of the molecule lies at 0.092 a.u. which the diatomic molecule can reach by absorbing $13\left(n_{\omega_{0}}\right)$ photons of $0 \rightarrow 1$ transition frequency $\left(\omega_{01}\right)$. The number of photons of frequency $\omega_{1}\left(n_{\omega_{1}}\right)$ required to reach dissociation threshold is 24 while $n_{\omega 2}=45, n_{\omega 3}=104, n_{\omega 4}=312$. The GA based optimization through the entire parameter space spanned by frequency ratio, intensities and phase angles lead to an evolving fitness function displayed in figure la. The increase of fitness occurs in steps and saturates after 45 generations. Search profiles for the field parameters are displayed in figures $1(\mathrm{~b}-$ d). The actual optimal intensities are $\varepsilon_{1}=0.00976$, $\varepsilon_{2}=0.0128, \varepsilon_{3}=0.01003$ and $\varepsilon_{3}=0.0099$ a.u. The phase angles are $\delta_{1}=3.2534, \delta_{2}=-4.448, \delta_{3}=$ 1.1606 and $\delta_{4}=-1.6261$, while the frequencies are in the ratio $45: 46: 47: 48$. The optimal frequencies are $\omega_{1}=0.00201, \omega_{2}=0.00193, \omega_{3}=0.00189$ and $\omega_{4}=0.00197$ a.u. These optimal parameters have been used for designing an optimal four component field for achieving the most facile photodissociation. The coupling between the field and the molecule is assumed to be linear. The dissociation

Table 1. Morse parameters of lithium hydride used in the calculation reported.

\begin{tabular}{lcccc}
\hline Molecule & $x_{e}$ (a.u.) & $D_{e}$ (a.u.) & $\beta$ & $\mu\left(\right.$ a.u. $\left.^{-1}\right)$ \\
\hline $\mathrm{LiH}$ & 3.01533 & 0.092 & 0.712 & 1604.8 \\
\hline
\end{tabular}



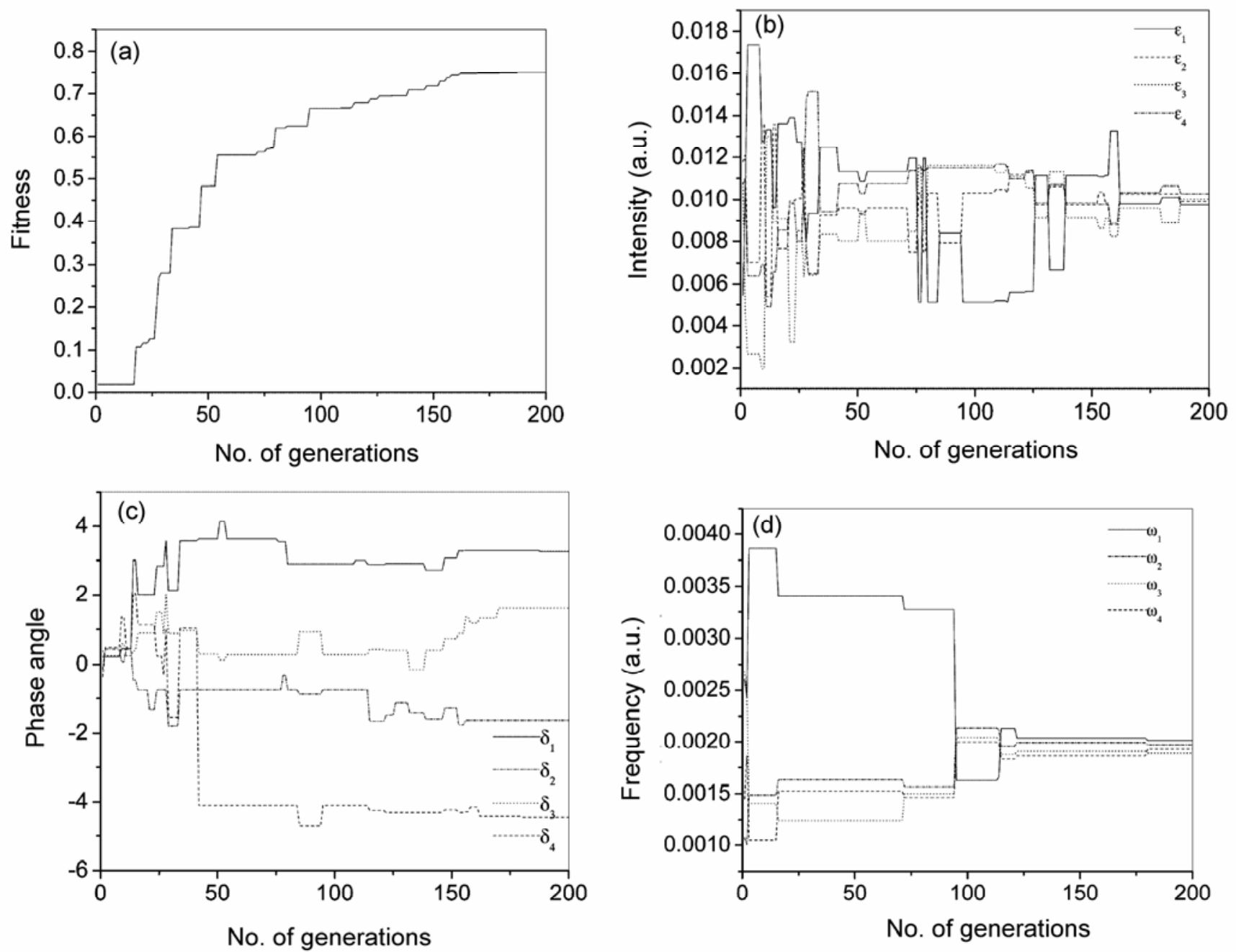

Figure 1. GA evolution profiles. (a) Plot of fitness function vs. number of generations, (b) frequencies vs number of generations, (c) phase vs number of generations, (d) frequency vs number of generations.

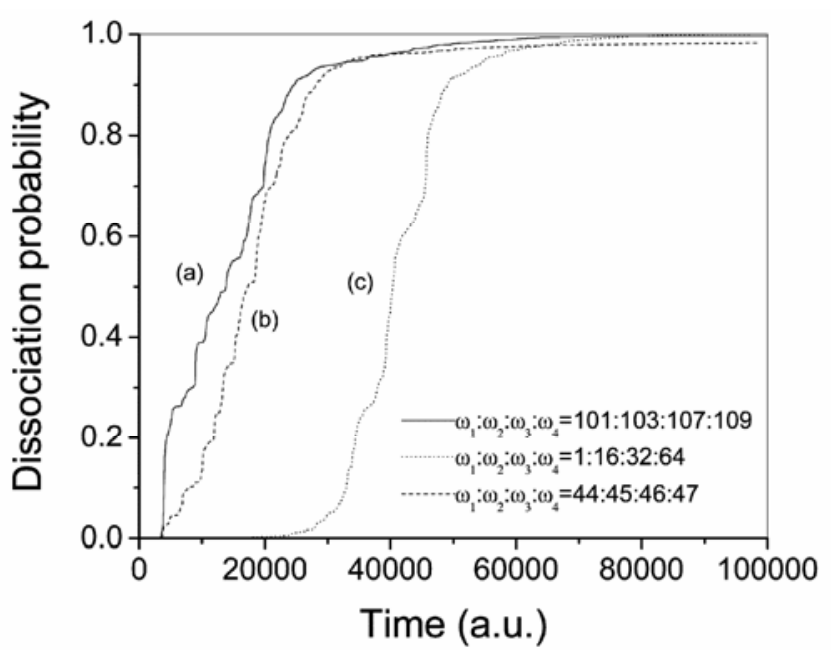

Figure 2. Plot of the dissociation probability using different frequency ratio with absorbing boundary condition. (a) for discretely optimized prime frequency, (b) for GA optimized parameters, (c) for discretely optimized nonprime frequencies. probability has been computed with absorbing boundary condition imposed for $x_{c} \geq 20$ a.u. on a grid of 30 a.u. and displayed in figure $2 \mathrm{a}$.

We have displayed in figures $2 b-c$ the growth of dissociation probability computed by us for a discretely optimized field with four prime and four non-prime frequencies, respectively. The result obtained with fully optimized four frequency field and only discretely optimized four prime frequency field are practically identical while result obtained with the field with four non-prime frequencies are manifestly worse. The fact that continuous optimization of the frequencies of the polychromatic field predicts three frequencies that are mutually prime and that the discrete optimization also led to a set of four prime frequencies of the polychromatic field indicate that there could be something fundamentally different in the prime and non-prime frequency cases. 

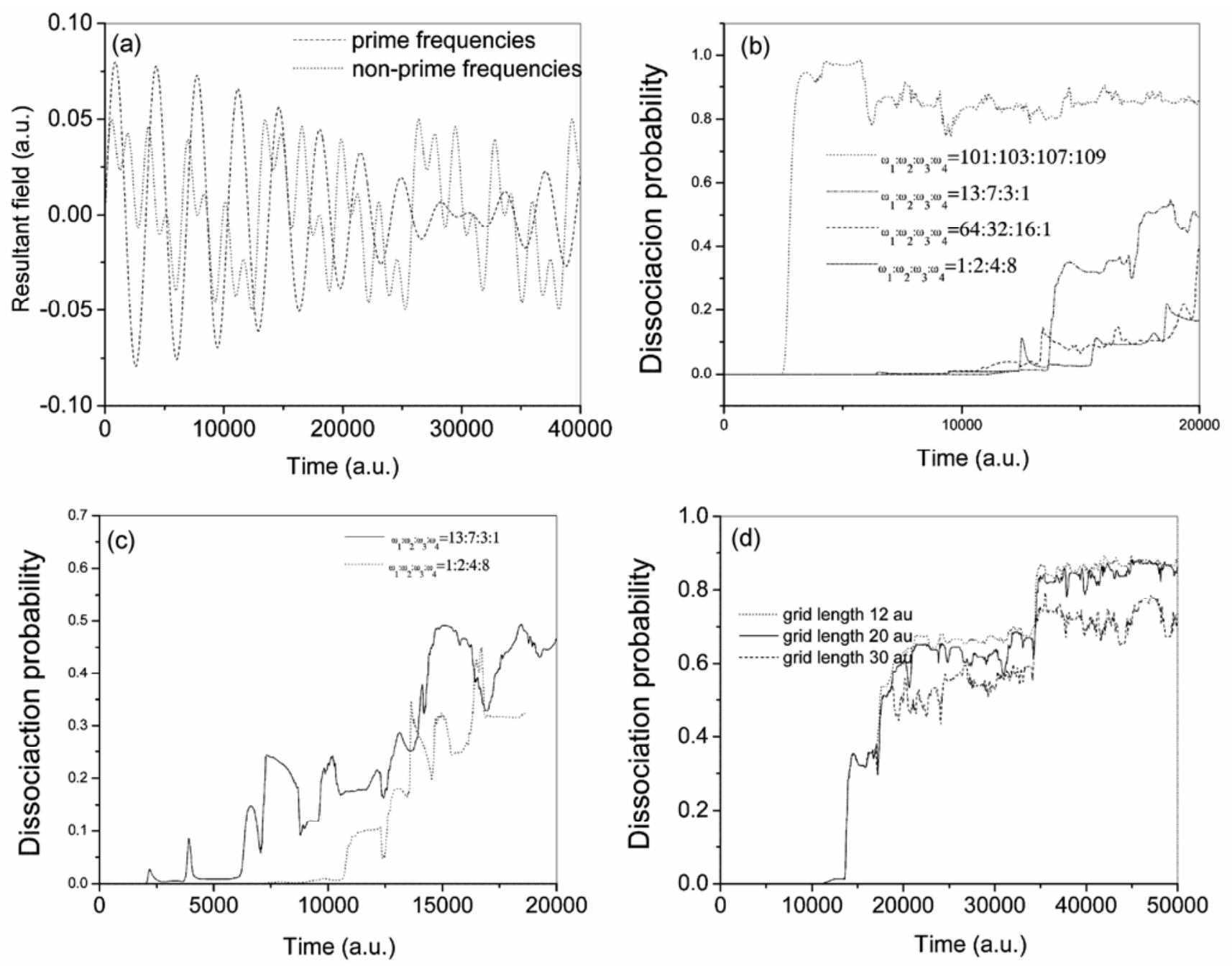

Figure 3. (a) Variation of the net field with time for four prime and four non-prime frequencies, (b) dissociation dynamics of $\mathrm{LiH}$ in a polychromatic field with effective intensity 0.02 a.u.; with no phase lag among the components, (c) when the relative phase lag among the component frequencies is $-\pi / 8$, (d) dissociation dynamics on grids of different length when no phase difference exist among the four components.

We have therefore investigated the problem further by using different sets of frequency ratios, two of them being in the ratio of primes and the other two sets being in non-prime number ratio $\left(\omega_{1}: \omega_{2}\right.$ : $\omega_{3}: \omega_{4}=1: 2: 4: 8, \quad \omega_{1}: \omega_{2}: \omega_{3}: \omega_{4}=1: 16: 32:$ 64). The exact frequencies of this four sets are $\left(\omega_{1}=0.003954, \omega_{2}=0.002129, \omega_{3}=0.0009125\right.$ a.u. and $\omega_{4}=0.000301$ a.u. $), \quad\left(\omega_{1}=0.001755, \quad \omega_{2}=\right.$ $0.001790, \omega_{3}=0.00185$ and $\omega_{4}=0.00189$ a.u. $)$, and $\left(\omega_{1}=0.0004866, \omega_{2}=0.000973, \omega_{3}=0.00194\right.$ $\omega_{4}=0.00389$ a.u. $)$ and $\left(\omega_{1}=0.00005488, \omega_{2}=\right.$ $0.001033, \omega_{3}=0.00206$ and $\omega_{4}=0.004134$ a.u.) respectively. In each case the same effective intensity and strength of linear molecule-field coupling have been used for comparison. The distinct difference in the performance of the prime and non-prime frequency polychromatic fields is curious. To probe it further, we have displayed typical profiles for the net fields as a function of time obtained by mixing prime and non-prime frequencies in figure 3(a). The nature of the time variation of the net field is widely different in the two cases and we anticipate that the difference in the response of the diatom to the two types of fields is partly due to the difference in the net field experienced by the molecule. The dissociation profile for each set is plotted in figure 3(b) for the zero phase cases $\delta=0$. Figure 3(c) shows the effect of introducing a relative phase difference of $\delta=(-\pi / 8)$ in a four prime as well as non-prime frequency polychromatic field. The most facile dissociation apparently takes place when $\omega_{1}: \omega_{2}: \omega_{3}$ : $\omega_{4}=101: 103: 107: 109$ and the least facile disso- 

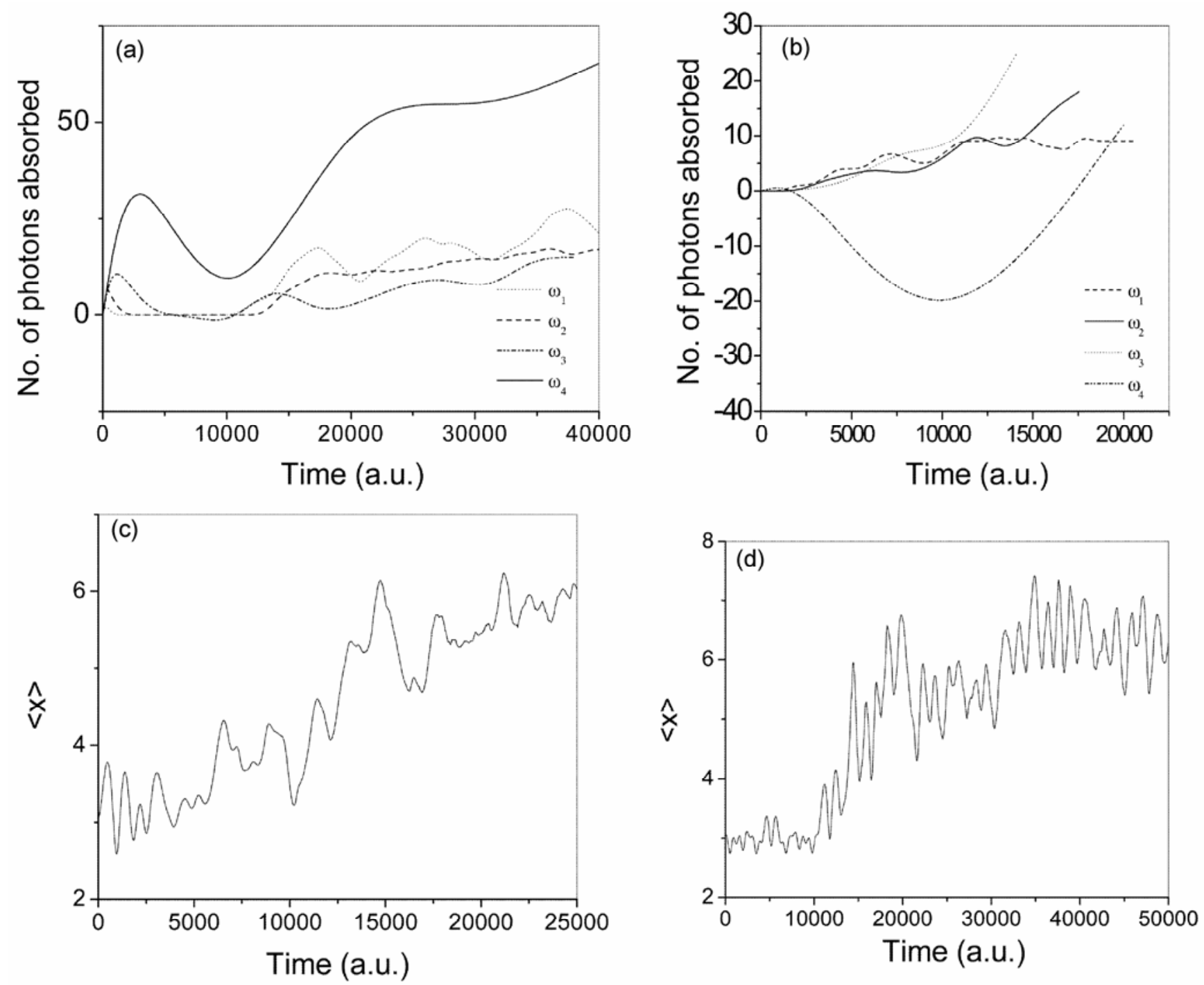

Figure 4. Plots of number of photons absorbed vs time for $\mathrm{LiH}$ undergoing dissociation in a polychromatic field with $\omega_{1}: \omega_{2}: \omega_{3}: \omega_{4}=1: 3: 7: 13$ and effective intensity $0 \cdot 02$ a.u. (a) with no phase lag, (b) with relative phase lag $-\pi / 8$. Plot of average Bond length vs time for $\mathrm{LiH}$ in a polychromatic field with $\omega_{1}: \omega_{2}: \omega_{3}: \omega_{4}=1: 3: 7: 13$ with effective intensity 0.02 a.u., (c) with relative phase lag of $-\pi / 8$, (d) with no phase lag.

ciation is achieved with figure 3(c) also shows the greater efficiency of the prime frequencies over their non-prime counterpart in the presence of a definite phase difference. Sirko and Koch's work ${ }^{16}$ revealed that the presence of rational frequency ratio is required for the existence of common resonances and controlled phase variations could affect the structures of such common resonances leading to the possibility of controlling ionization probability of $\mathrm{H}$ atom. Present results indicate the possible existence of similar features in multi-photon dissociation of diatoms.

When the nature of the coupling between the oscillator and the radiation field is changed to a Gaussian type of coupling $\left(V_{1}(x, t)\right.$ of $\left.(4)\right)$ some quantitative change is observed in the time dependence of the computed dissociation probability. If $f(x)$ of (3) is a Gaussian with $b=3 \cdot 15$ the molecule is found to undergo dissociation at a higher threshold intensity compared to the linear coupling case $(0.04$ a.u. visa-vis to 0.02 a.u. in the linear case). Table 2 shows the comparative features of the dissociation characteristics observed with linear and Gaussian coupling, respectively keeping the other parameters of the model unchanged. Gaussian coupling lowers both the dissociation probability and dissociation rate, although the profiles may not reveal any qualitative differences. 

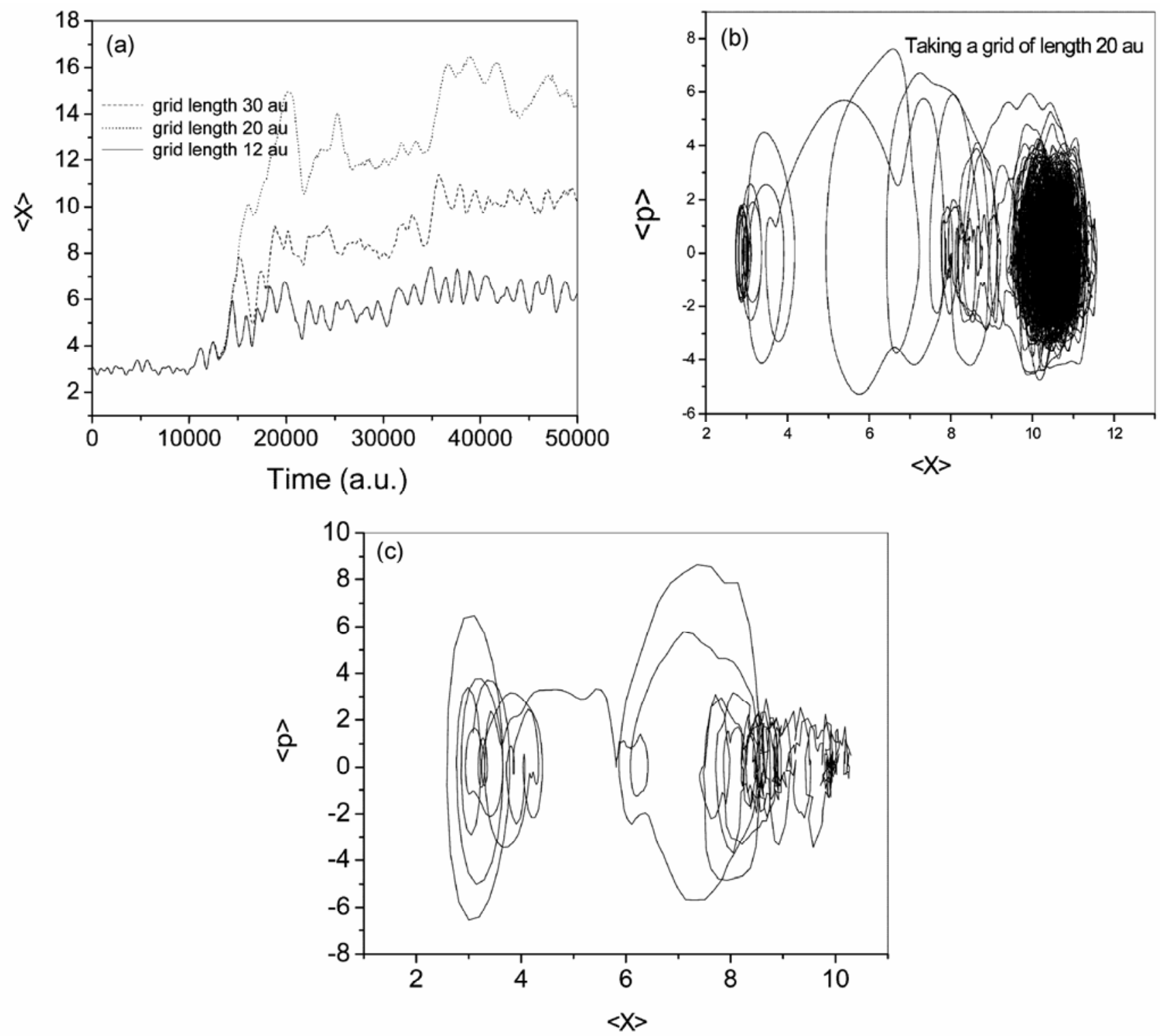

Figure 5. (a) Plots of average bond length against time ongrids of different length for frequency ratio $\omega_{1}: \omega_{2}: \omega_{3}: \omega_{4}=1: 3: 7: 13$ and effective intensity of 0.02 a.u. without any phase lag among the components. Quantum phase space structures of $\mathrm{LiH}$ undergoing dissociation in a polychromatic field with $\omega_{1}: \omega_{2}: \omega_{3}: \omega_{4}=1: 3: 7: 13$ with effective intensity 0.02 a.u., (b) with no phase lag, (c) with a phase lag $-\pi / 8$ among the components.

Table 2. Average dissociation rate constant and dissociation probability of lithium hydride for linear and Gaussian type of coupling between the molecule and the field.

\begin{tabular}{lccccc}
\hline Molecule & $\begin{array}{c}\text { Intensity } \\
\text { (a.u.) }\end{array}$ & $\begin{array}{c}\text { Frequency } \\
\text { ratio }\end{array}$ & $\begin{array}{c}\text { Nature of } \\
\text { coupling }\end{array}$ & $\begin{array}{c}\text { Predicted rate } \\
\text { constant (averaged) (a.u) })^{-1}\end{array}$ & $\begin{array}{c}\text { Dissociation } \\
\text { probability at } t=T\end{array}$ \\
\hline $\mathrm{LiH}$ & $0 \cdot 02$ & $13: 7: 3: 1$ & Harmonic & $0 \cdot 0009$ & $0 \cdot 73(50000)$ \\
$\mathrm{LiH}$ & $0 \cdot 05$ & $13: 7: 3: 1$ & Gaussian & $0 \cdot 00013$ & $0.63(50000)$ \\
$\mathrm{LiH}$ & 0.04 & $13: 7: 3: 1$ & Gaussian & $0 \cdot 000035$ & $0.27(350000)$ \\
\hline
\end{tabular}

The qualitative features of the response also do not depend much on the length of the grid. We have computed the dissociation profiles of $\mathrm{LiH}$, with three different grids of lengths $L$ equal to 12 a.u., 
20 a.u. and 30 a.u., respectively; and in each case, we have obtained almost similar dissociation profiles. These profiles are displayed in the figure 3(d) for comparison.

We have analysed the time and frequency resolved pattern figures $4(a-b)$ of the gain in number of photons by the diatomic molecule in the 'prime frequencies' scenario both when there is a phase difference (figure 4a) and there is none (figures $4 b$ ). The growth rate of the number of photons absorbed $\left(n_{\omega}\right)$ is more or less the same for the first three frequencies i.e. $\left(n_{\omega_{1}}\right),\left(n_{\omega_{2}}\right)$ and $\left(n_{\omega_{3}}\right)$ are nearly equal. $\left(n_{\omega_{4}}\right)$, however registers an initial decrease, passes through a minimum and then increases again to match $\omega_{1}$ or $\omega_{2}$, when $\delta_{i}=-\pi / 8$ (figure $4 \mathrm{a}$ ). If the phase difference is made to disappear, $\left(n_{\omega_{4}}\right)$ shows sharp rise after passing through a minimum and $\left(n_{\omega_{4}}>>n_{\omega_{1}}\right)$ or $\left(n_{\omega_{2}}\right)$ or $\left(n_{\omega_{3}}\right)$ in the long time limit (figure $4 \mathrm{~b}$ ).

The evolution of bond length with time for $\delta_{i}=$ $-\pi / 8$ shows almost linear increase with oscillations while for $\delta_{i}=0$ there is saturation as $t \rightarrow \infty$ (figures $4 \mathrm{c}-\mathrm{d})$. In either case, absorbing boundary condition has not been imposed. The effect of the length of the grid used on the observed bond stretching dynamics is investigated in figures 5a. In each case there is an initial linear increase followed by a saturation near $\langle x\rangle=L / 2$ where $L$ is the length of the corresponding grid. All other features of the bond stretching dynamics are qualitatively similar. We have followed how $\langle x(t)\rangle,\left\langle p_{x}(t)\right\rangle$ evolve during the dissociation process where $\langle a\rangle$ indicates the ensemble average of $a$. The $\left\langle p_{x}(t)\right\rangle$ versus $\langle x(t)\rangle$ plots may be thought of as providing a quantum phase space picture of the process.

The 'quantum phase space' structures for $L=$ 20 a.u. are displayed in figures $5 \mathrm{~b}-\mathrm{c}$. A localization is seen to occur at $\langle x\rangle=10$ a.u. The presence of phase difference affects the phase space structures and the localization around $\langle x\rangle=10$ a.u. seen to be more pronounced in the zero phase difference $\left(\delta_{i}=0\right)$ case. The other features are quite identical for $\delta=0$ (figure $5 \mathrm{~b}$ ) and $\delta=-\pi / 8$ (figure $5 \mathrm{c}$ ). We tend to conclude therefore that a suitably tuned four component field could lead to faster and more complete dissociation of a diatomic molecule and a good way to tune would be to select prime frequency ratios or use GA to predict the appropriate frequencies, phases and intensities. The frequency resolved energy gain pattern is complex but the underlying bond stretching dynamics seems to follow the classical pattern.

\section{Conclusion}

It appears that above a threshold of effective intensity poly-chromatic fields with four incommensurate non-resonant low frequencies could lead to facile photo-dissociation of diatomic-molecules with high average rate and percentage of dissociation. The quantum phase space structures of the dissociating diatoms reveal pictures that are reminiscent of the classical non-linear dynamical bond breaking dynamics. Relative phase differences do not modify the gross features of phase space structure although they affect dissociation rates.

\section{Acknowledgements}

One of the authors (SG) would like to thank the Council of Scientific and Industrial Research (CSIR), New Delhi, Government of India for the award of Senior Research Fellowship. R S and S P B thank the Department of Science and Technology (DST), Government of India, New Delhi for a generous research grant.

\section{References}

1. Mittleman M H 1982 Introduction to the theory of laser-atom interaction (New York: Plenum)

2. Some theoretical aspects of infra-red lasers pumped by electronic-to-vibrational energy transfer: Atomic and molecular process with short intense laser pulses (ed.) Brandrauk (New York: Plenum) 1988 NATO ASI series B 171461

3. Bandrauk A D and Wallancevol S C 1992 Coherent phenomenon in atoms and molecules in laser fields (New York: Plenum) NATO ASI, series B: 28765

4. Aupanel E E, Jean Marc Gauthier and Bandrauk A D 1993 Phys. Rev. A48 2145

5. Chelkowski S and Bandrauk A D 1990 Phys. Rev. A41 6480

6. Atwood D, Soni A and Wyler D 1990 Phys. Rev. Let. $\mathbf{6 5} 2335$

7. Goggin M E and Milonni P W 1988 Phys. Rev. A37 796

8. Breuer H P, Dietz K and Holthaus M 1988 Z. Phys. D8 349

9. Bloembergen N and Zewail A H $1984 J$. Phys. Chem. 885459

10. Letokhov V S 1983 Non-linear laser chemistry (Berlin: Springer)

11. Shapiro M and Brumer P 2003 Rep. Prog. Phys. 66 859

12. Ehlotzky F 2001 Phys. Rep. 345175

13. Constantoudis V and Nicolaides C A 1997 Phys. Rev. A55 1325 
14. Constantoudis V and Nicolaides C A $2005 \mathrm{~J}$. Chem. Phys. 12284118

15. Schumacher D W, Weiche F, Muller H G and Bucksbaum H P 1994 Phys. Rev. Lett. 73344

16. Sirko L and Koch P M 2002 Phys. Rev. Lett. 89 274101

17. Holland J H 1975 Adaptation in natural and artificial systems (Ann Arbar, MI: University of Michigan Press)

18. Goldberg D E 1989 Genetic algorithms in search, optimisation and machine learning (Reading, MA: Addision Wesley)

19. Michaelwicz Z 1995 Genetic Algorithms + Data Structure $=$ Evolution Programs $\}$ (London, UK: Springer) 3rd edn

20. Saha R, Chaudhury P and Bhattacharyya S P 2001 Phys. Lett. A291 397
21. Adhikari S, Dutta P and Bhattacharyya S P 1992 Chem. Phys. Lett. 199574

22. Adhikari S and Bhattacharyya S P 1992 Phys. Lett. A172 155

23. Adhikari S, Dutta P and Bhattacharyya S P 1996 Chem Phys. 206315

24. Marston C C and Baliant Kurti G B 1989 J. Chem. Phys. 913571

25. Balint Kurti G B, Ward C L and Marton C C 1991 Comput. Phys. Commun. 67285

26. Dirac P A M 1993 Proc. Cambridge Phys Soc. 26 376

27. Frenkel J 1934 Wave mechanics (Oxford: Oxford University Press)

28. Ghosh M, Sharma R and Bhattacharyya S P 2007 Chem. Phys. Lett. 449165

29. Erkol H and Demiralp E 2007 Phys. Lett. A365 55 